\title{
Análise dos gastos com diagnóstico por imagem no Brasil
}

\section{Analysis of expenses with diagnostic imaging in Brazil}

\author{
Graziela Liebel' ${ }^{1}$, , Patrick Vieira Dias', lone Jayce Ceola Schneider², Antônio Reis de Sá Junior², \\ Agenor Hentz², Clécio da Silva Ferreira', Alfredo Chaoubah' \\ ${ }^{1}$ Programa de pós-graduação em Saúde Coletiva, Universidade Federal de Juiz de Fora (UFJF) - Juiz de Fora (MG), Brasil. \\ ${ }^{2}$ Departamento Ciências da Saúde, Universidade Federal de Santa Catarina (UFSC) - Florianópolis (SC), Brasil.
}

Como citar: Liebel G, Dias PV, Schneider IJC, Sá Junior AR, Hentz A, Ferreira CS, Chaoubah A. Análise dos gastos com diagnóstico por imagem no Brasil. Cad Saúde Colet, 2021;29(3):453-463. https://doi.org/10.1590/1414-462X202129030397

\section{Resumo}

Introdução: Os gastos em saúde têm sido uma preocupação mundial diante da escassez de recursos financeiros para o atendimento das demandas em saúde. Objetivo: Analisar o gasto per capita com diagnóstico por imagem de alta complexidade e associação com variáveis sociodemográficas e estruturais no Brasil. Método: Estudo ecológico observacional dos gastos com diagnóstico por imagem utilizando os registros do Sistema de Informação do Sistema Único de Saúde (SUS), com análise de regressão com modelos generalizados de efeitos mistos, de 2008 a 2017, por macrorregiões de saúde, de acordo com as variáveis: PIB per capita, população, taxa de médicos, taxa de equipamentos em uso no SUS e cobertura pela Estratégia Saúde da Família (ESF). Resultados: Na análise descritiva dos dados, foi observado aumento dos gastos com diagnóstico por imagem de alta complexidade. Foi identificada correlação positiva entre o gasto e as variáveis taxa de médicos, taxa de equipamentos em uso no SUS e PIB, bem como correlação negativa com a cobertura pela ESF. Conclusão: A relação entre ampliação da cobertura pela ESF e diminuição no gasto com diagnóstico por imagem de alta complexidade se apresentou como importante indicador no direcionamento das políticas públicas e de recursos. Assim, macrorregiões com maiores taxas do PIB e de médicos tendem a ter gastos mais elevados com diagnóstico por imagem de alta complexidade.

Palavras-chave: Sistema Único de Saúde; Diagnóstico por imagem; Financiamento da Assistência à Saúde; Sistemas de Informação em Saúde.

\begin{abstract}
Background: Health expenditures have become a global concern given the scarcity of financial resources to meet health demands. Objective: To analyze the trend of per capita expenditure with high-complexity diagnostic imaging and its association with sociodemographic and structural variables in Brazil. Method: Observational, ecological study on diagnostic imaging expenditures using the Unified Health System (SUS) information system records, through regression analysis with Generalized Mixed Effects Models, from 2008 to 2017 , by health macro-regions, according to the following variables: GDP per capita, population, rate of physicians, rate of equipment in use at SUS, and coverage by the Family Health Strategy (FHS). Results: Descriptive analysis of the data showed an increase in expenses with high-complexity diagnostic imaging. Expenditure was positively correlated with rate of physicians, rate of equipment in use in SUS and GDP per capita, and negatively correlated with FHS coverage. Conclusion: The relationship between expansion of coverage by the FHS and decreased expenses with high-complexity diagnostic imaging was an important indicator to direct public policies and allocate resources. Thus, macro-regions with higher GDP per capita and rate of physicians tend to have greater expenses with high-complexity diagnostic imaging.
\end{abstract}

Keywords: Unified Health System; Diagnostic imaging; Health Care Financing; Health Information Systems.

Este é um artigo publicado em acesso aberto (Open Access) sob a licença Creative Commons Attribution, que permite uso, distribuição e reprodução em qualquer meio, sem restrições desde que o trabalho original seja corretamente citado.
O estudo foi realizado no UFJF Faculdade de Medicina Dados secundários advindo do DataSus - Juiz de Fora (MG), Brasil.

Correspondência: Graziela Liebel. E-mail: grazielaliebel_fono@hotmail.com

Fonte de financiamento: nenhuma.

Conflito de interesses: nada a declarar.

Recebido em: Set. 25, 2019. Aprovado em: Jul. 20, 2020 


\section{INTRODUÇÃO}

Os gastos em saúde têm sido uma preocupação mundial diante da escassez de recursos financeiros para o atendimento das demandas em saúde. Desde o final dos anos 1990, o uso de modalidades avançadas de diagnóstico por imagem aumentou significativamente nos Estados Unidos e superou o aumento dos gastos médicos em geral. Os gastos com os serviços médicos cresceram $31 \%$, enquanto os diagnósticos por imagem aumentaram $62 \%$.

Ainda nos anos 1990, a Inglaterra registrou aumento do número total de diagnósticos por imagem em $40 \%$, o que representou crescimento médio de $3,4 \%$ ao ano. Durante esse período, o volume de exames de ressonância magnética aumentou em $220 \%$, e de tomografias computadorizadas, em $160 \%$, o que gerou crescimento médio por ano de $12,3 \%$ e $10,1 \%$, respectivamente ${ }^{2}$.

Entre os fatores discutidos para explicar o aumento mundial do número de procedimentos de imagem e a consequente elevação dos custos em saúde, destacam-se: o avanço da tecnologia, a ampliação do acesso e da demanda por pacientes e médicos, além do crescimento da oferta de serviços de atenção à saúde, especialmente daqueles de pagamento por produção $0^{3,4}$. Essa realidade se torna um problema diante do quadro comum de escassez de recursos econômicos para os sistemas de saúde universais, visto que há necessidade de se ofertar essa tecnologia para a população da forma mais racional e equitativa possível ${ }^{5,6}$. Nesse contexto, é crucial que o processo de tomada de decisão seja eficiente e responsável, priorizando a gestão do cuidado em saúde ${ }^{4,5}$.

Os sistemas de saúde em muitos países em desenvolvimento, como o Brasil, enfrentam grandes desafios para atender à demanda de padrões de saúde e tecnologia do século XXI. Nesses países, os recursos disponíveis permanecem abaixo do que as nações desenvolvidas investiram há 30 anos. O Brasil tem investido apenas US\$ 1,043 per capita anualmente em saúde, o que equivale a apenas $31 \%$ da média da Organização para a Cooperação e Desenvolvimento Econômico $(\mathrm{OCDE})^{7}$. Priorizar um modelo que respeite os princípios éticos e favoreça a justiça distributiva em saúde é essencial para que se alcancem a equidade e a eficiência desejadas na área da saúde no Brasil ${ }^{5,6}$.

O estudo de Dilélio et al. ${ }^{8}$ apontou que os padrões de utilização de serviços de saúde são socialmente determinados pelo resultado da oferta, das características sociodemográficas e do perfil de saúde dos usuários. Dessa forma, é possível que exista uma relação importante entre os gastos federais com procedimentos de diagnóstico por imagem nas macrorregiões de saúde brasileiras e as variáveis demográficas, socioeconômicas e estruturais. Assim, o objetivo desta pesquisa foi analisar as variáveis que possuem maior influência sobre os gastos com diagnóstico por imagem de alta complexidade no período de 2008 a 2017. Este estudo pode potencializar a elaboração de estratégias que sejam custo-efetivas com a reestruturação da contratação e execução desses serviços e contribuir para a elaboração das políticas públicas de saúde no Brasil.

\section{MÉTODO}

\section{Desenho do estudo}

Este é um estudo ecológico e analítico, abrangendo todas as 104 macrorregiões de saúde do Brasil. A regionalização tem por objetivo organizar a atenção à saúde de maneira que atenda a população em todos os níveis de atenção: primário, secundário e terciário. Foi empregada a análise dos valores gastos com diagnóstico por imagem de alta complexidade nos anos de 2008 a 2017 no Sistema Único de Saúde (SUS).

Foi utilizada a base de dados administrativos dos seguintes Sistemas de Informação do Departamento de Informática do Sistema Único de Saúde (Datasus): Sistema de Informações Ambulatoriais do SUS (SIA/SUS) - assistência à saúde; Sistema de Informação Hospitalar (SIH) - serviços profissionais; Sistema do Cadastro Nacional de Estabelecimentos em Saúde (SCNES) - rede assistencial; além dos Censos Demográficos de 2000 e 2010, do Instituto Brasileiro de Geografia e Estatística (IBGE) ${ }^{9}$. 
Os procedimentos de diagnóstico por imagem de alta complexidade são controlados por meio de autorização de procedimentos de alta complexidade (APAC), subsidiados com recursos do limite financeiro de média e alta complexidade (MAC) ambulatorial e hospitalar ${ }^{10}$. Os valores das APACs integram o conjunto de dados do SIA/SUS, que é responsável por todos os serviços e procedimentos ambulatoriais, como consultas médicas e exames diagnósticos por imagem, procedimentos clínicos e cirúrgicos, fisioterapia, acupuntura, reabilitação e outros procedimentos registrados no Sistema de Gerenciamento da Tabela de Procedimentos, Medicamentos, Órteses, Próteses e Materiais Especiais (SIGTAP) ${ }^{10}$.

Os dados de custos dos procedimentos de diagnóstico por imagem de alta complexidade foram obtidos por meio do SIA/SUS e SIH, com o valor aprovado em reais. Os valores financeiros foram atualizados utilizando o Índice de Preços ao Consumidor Amplo (IPCA) do IBGE, acumulado de 2018.

Foram estratificados do Datasus os procedimentos com finalidade diagnóstica denominado grupo $02^{11}$, levando em conta os subgrupos de maior relevância financeira: radiodiagnóstico, exames ultrassonográficos, tomografia computadorizada, ressonância magnética, medicina nuclear in vivo e radiologia intervencionista, combinados em uma única categoria de diagnóstico por imagem, a qual foi relativizada pela população das macrorregiões de saúde, sendo uma variável de interesse e denominada como gasto per capita com diagnóstico por imagem de alta complexidade.

Considerando que os dados de gastos foram extraídos de uma base de dados administrativa, que não registra todos os custos de procedimentos realizados, foram assumidos os valores registrados no SIA/SUS e SIH como proxy do gasto com diagnóstico por imagem no Brasil para a análise dos dados.

Foram consideradas as características de população, produto interno bruto (PIB) e estrutura dos serviços de saúde de alta complexidade, com a finalidade de avaliar a relação ao longo do tempo de tais variáveis com o gasto per capita com diagnóstico por imagem de alta complexidade.

As características das variáveis independentes por macrorregião de saúde foram: população e PIB per capita por mil reais - este obtido por meio de agregação do PIB municipal por macrorregião de saúde.

As características de estrutura dos serviços de saúde de alta complexidade foram: equipamentos de imagem em uso no SUS, número de médicos por mil habitantes e cobertura populacional pela Estratégia Saúde da Família (ESF) ${ }^{12}$ - esta última foi obtida por meio da fórmula Equação (1):

$n^{\circ}$ de equipes $S f \times 3000$ pessoas $\left(n^{\circ}\right.$ médio estimado de pessoas cadastradas por equipe $) \times 100$ total da população do município ( população macrorregiões de saúde)

Para todos os indicadores, foram criadas as taxas brutas a partir da divisão entre a frequência absoluta da característica e a população do local para cada ano. O software TABWIN, versão 1.4.1, foi utilizado para a extração e o processamento de dados de saúde.

Inicialmente, foi realizada análise descritiva das variáveis nos 10 anos, obtendo-se a média e o desvio-padrão (DP), com a aplicação do teste de normalidade Shapiro-Wilk para as variáveis de interesse da amostra e de correlação de Pearson com coeficiente $p>0,005$. Para a análise desses dados, foi usado o software SPSS Statistics, versão 21.0 (Chicago, IL 60606, Estados Unidos).

Para a análise de regressão, foram utilizados modelos lineares generalizados com efeitos mistos (GLMM) por meio do pacote Ime4 software $\mathrm{R}^{13}$, na versão 3.5.3, função GLMER, para a identificação dos fatores associados aos gastos com imagem, modelo obtido para os dados padronizados. Foram utilizadas as distribuições da família exponencial (FE) para dados positivos (gasto), isto é, gama (e suas funções de ligação), normal inversa (e suas funções de ligação) e normal com ligação log. Além disso, foram testadas e selecionadas todas as interações duas a duas entre as variáveis explicativas numéricas.

O GLMM Equação (2) considerado foi:

$$
\left\{\begin{aligned}
E\left(y_{i j} \mid t_{j}\right) & =\mu_{i j}, i=1, \ldots, n ; j=1, \ldots, t \\
g\left(\mu_{i j}\right) & =\beta_{0}+\sum_{k=1}^{p} \beta_{k} X_{i j k}+t_{j},
\end{aligned}\right.
$$


em que: $n=104$ é o número de observações (macrorregiões); $t_{j}$ é o efeito aleatório dado pelo ano no tempo $j(\mathrm{t}=10)$; $p$ é número de variáveis explicativas; $\beta_{0}$ é o parâmetro intercepto; e $g\left(\mu_{i j}\right)$ é a função de ligação. A variável resposta $y_{i j}$ pertence à $\mathrm{FE}\left(\mu_{i j}, \varnothing\right), \operatorname{com} \varnothing=\left(\varnothing_{\boldsymbol{t}}, \varnothing_{\boldsymbol{e}}\right)$, em que $\varnothing_{\boldsymbol{e}}$ é o parâmetro de dispersão da distribuição de $y_{i j} \mid t_{j}$ e $\varnothing_{t}$ é a variância do efeito aleatório $\left(t_{j} \sim N\left(0, \varnothing_{t}\right)\right)$.

Após o ajuste de cada distribuição e função de ligação, o melhor modelo ajustado foi o da distribuição gama, com ligação log Equação (3), isto é,

$\log \left(\mu_{i j}\right)=\beta_{0}+\sum_{k=1}^{p} \beta_{k} X_{i j k}+t_{j}$

Assim, $\mu_{i j}=e^{\beta_{0}+\sum_{k=1}^{p} \beta_{k} X_{i j k}+t_{j}}$ e mantidas as demais variáveis constantes, o aumento de uma unidade da variável $X_{k}$ aumenta em $e^{\beta_{k}}$ o valor esperado da variável resposta. Ou, simplesmente, se $\beta_{k}>0\left(\beta_{k}<0\right)$, é esperado um aumento (diminuição) na variável resposta quando $X_{k}$ aumenta.

Este estudo foi aprovado pelo Comitê de Ética em Pesquisa em Seres Humanos da Universidade Federal de Juiz de Fora, sob protocolo no 1.942 .507 de 2017.

\section{RESULTADOS}

O gasto médio per capita com diagnóstico por imagem de alta complexidade nas macrorregiões de saúde, em uma análise descritiva da população total do estudo, apresentou elevação ao longo do período avaliado (Figura 1), partindo de 2008 com $R \$ 3,26$ ( $D P=1,94$ ), seguindo com queda em 2015, em relação a 2014, com $R \$ 5,46(D P=2,46)$, e alta novamente em 2017 , com $\mathrm{R} \$ 5,81(\mathrm{DP}=3,08)$. Já a média da população estimada das macrorregiões de saúde cresceu discretamente ao longo de 10 anos, de 1.823.200,13 habitantes $(D P=2.135 .049,90)$ para 1.996.739,70 habitantes $(\mathrm{DP}=2.302 .490,20)($ Tabela 1$)$.

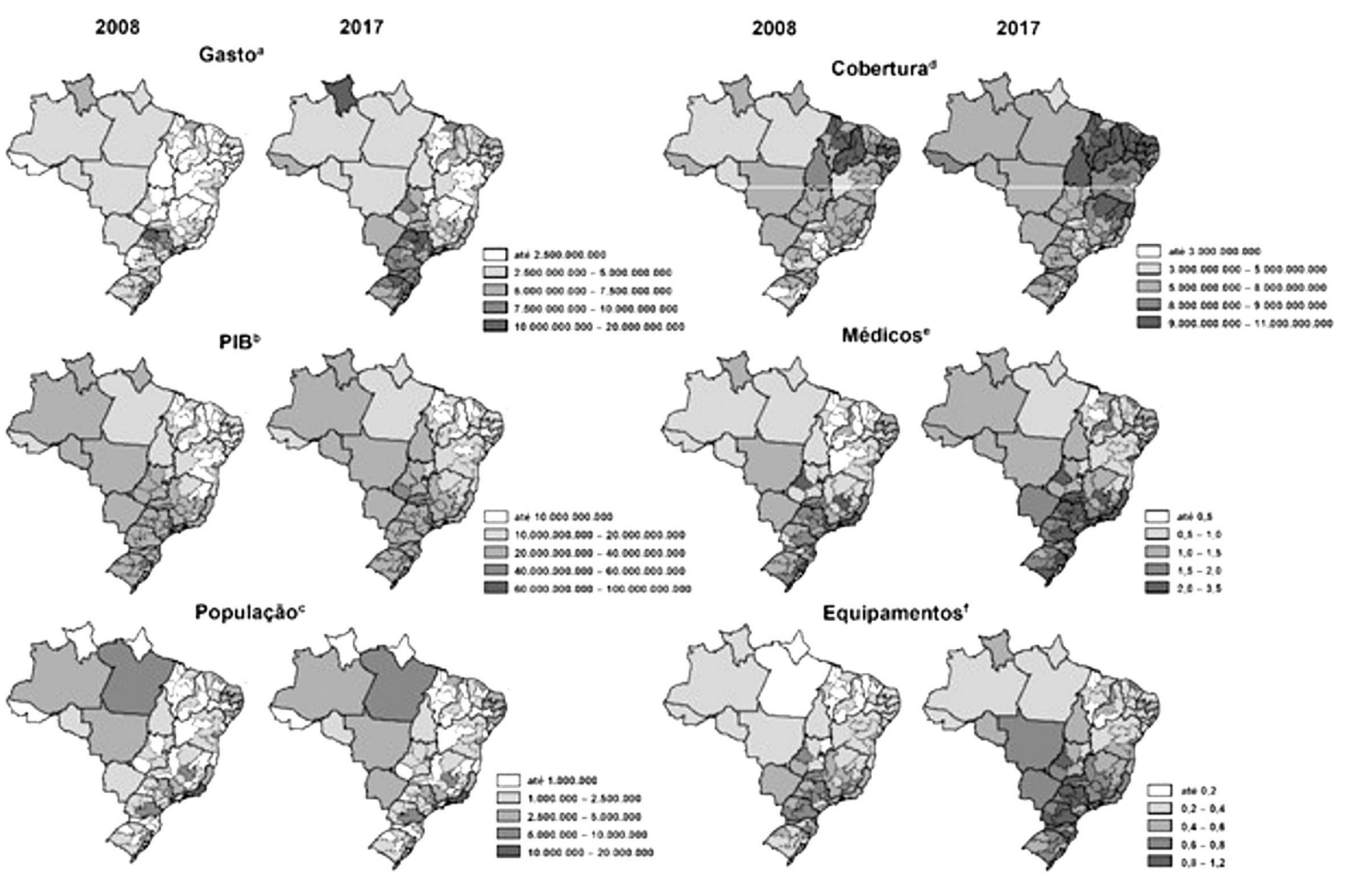

Figura 1. Mapas de evolução das variáveis nas macrorregiões de saúde do Brasil nos anos de 2008 e 2017. Fonte: Datasus ${ }^{14}$ e IBGE ${ }^{33}$. Elaboração dos autores Departamento de Informática do SUS. Notas técnicas. Origem dos dados e descrição das variáveis disponíveis para a tabulação. (a) Gasto per capita com diagnóstico por imagem; (b) Produto Interno Bruto; (c) População da macrorregião de saúde; (d) Cobertura da Estratégia Saúde da Família (ESF); (e) Médicos por mil habitantes; (f) Equipamentos de imagem para cada mil habitantes. 
Tabela 1. Caracterização das variáveis estudadas por macrorregião de saúde, Brasil, 2008 a 2017

\begin{tabular}{|c|c|c|c|c|c|c|c|c|c|c|}
\hline & \multicolumn{10}{|c|}{ Média (desvio-padrão) } \\
\hline & 2008 & 2009 & 2010 & 2011 & 2012 & 2013 & 2014 & 2015 & 2016 & 2017 \\
\hline $\begin{array}{l}\text { População } \\
\text { estimada (em } \\
\text { milhão) }\end{array}$ & $1,82(2,13)$ & $\begin{array}{c}1,84 \\
(2,15)\end{array}$ & $\begin{array}{c}1,83 \\
(2,15)\end{array}$ & $\begin{array}{c}1,84 \\
(2,17)\end{array}$ & $\begin{array}{c}1,86 \\
(2,18)\end{array}$ & $\begin{array}{c}1,93 \\
(2,24)\end{array}$ & $\begin{array}{c}1,94 \\
(2,25)\end{array}$ & $\begin{array}{c}1,96 \\
(2,27)\end{array}$ & $\begin{array}{c}1,98 \\
(2,28)\end{array}$ & $\begin{array}{c}1,99 \\
(2,30)\end{array}$ \\
\hline $\begin{array}{l}\text { Gasto per } \\
\text { capita com } \\
\text { diagnóstico } \\
\text { por imagem }\end{array}$ & $\begin{array}{c}3,26 \\
(1,94)\end{array}$ & $\begin{array}{c}3,80 \\
(2,16)\end{array}$ & $\begin{array}{c}4,35 \\
(2,49)\end{array}$ & $\begin{array}{c}4,77 \\
(2,74)\end{array}$ & $\begin{array}{l}5,03 \\
(2,74)\end{array}$ & $\begin{array}{c}5,23 \\
(2,77)\end{array}$ & $\begin{array}{l}5,66 \\
(2,92)\end{array}$ & $\begin{array}{c}5,46 \\
(2,97)\end{array}$ & $\begin{array}{c}5,62 \\
(2,93)\end{array}$ & $\begin{array}{c}5,81 \\
(3,08)\end{array}$ \\
\hline $\begin{array}{l}\text { Produto } \\
\text { interno bruto* }\end{array}$ & $\begin{array}{c}24,23 \\
(14,81)\end{array}$ & $\begin{array}{l}14,27 \\
(8,71)\end{array}$ & $\begin{array}{c}16,63 \\
(10,24)\end{array}$ & $\begin{array}{c}18,63 \\
(11,24)\end{array}$ & $\begin{array}{c}20,46 \\
(12,06)\end{array}$ & $\begin{array}{c}22,01 \\
(12,73)\end{array}$ & $\begin{array}{c}23,93 \\
(13,79)\end{array}$ & $\begin{array}{c}24,80 \\
(14,12)\end{array}$ & $\begin{array}{c}25,9 \\
(14,73)\end{array}$ & $\begin{array}{c}25,95 \\
(14,69)\end{array}$ \\
\hline $\begin{array}{l}\text { Equipamento } \\
\text { de imagem* }\end{array}$ & $\begin{array}{c}0,38 \\
(0,19)\end{array}$ & $\begin{array}{c}0,41 \\
(0,20)\end{array}$ & $\begin{array}{c}0,44 \\
(0,21)\end{array}$ & $\begin{array}{c}0,46 \\
(0,22)\end{array}$ & $\begin{array}{c}0,49 \\
(0,22)\end{array}$ & $\begin{array}{c}0,49 \\
(0,46)\end{array}$ & $\begin{array}{c}0,51 \\
(0,22)\end{array}$ & $\begin{array}{c}0,52 \\
(0,22)\end{array}$ & $\begin{array}{c}0,51 \\
(0,22)\end{array}$ & $\begin{array}{c}0,55 \\
(0,23)\end{array}$ \\
\hline Médicos* & $\begin{array}{c}1,12 \\
(0,55)\end{array}$ & $\begin{array}{c}1,17 \\
(0,57)\end{array}$ & $\begin{array}{c}1,22 \\
(0,60)\end{array}$ & $\begin{array}{c}1,20 \\
(0,60)\end{array}$ & $\begin{array}{c}1,25 \\
(0,63)\end{array}$ & $\begin{array}{c}1,28 \\
(0,61)\end{array}$ & $\begin{array}{c}1,28 \\
(0,61)\end{array}$ & $\begin{array}{c}1,39 \\
(0,63)\end{array}$ & $\begin{array}{c}1,44 \\
(0,66)\end{array}$ & $\begin{array}{c}1,51 \\
(0,68)\end{array}$ \\
\hline $\begin{array}{l}\text { \% de } \\
\text { cobertura } \\
\text { Estratégia } \\
\text { Saúde da } \\
\text { Família }\end{array}$ & $\begin{array}{c}57,94 \\
(24,37)\end{array}$ & $\begin{array}{c}59,49 \\
(25,06)\end{array}$ & $\begin{array}{c}61,64 \\
(25,14)\end{array}$ & $\begin{array}{c}62,15 \\
(24,78)\end{array}$ & $\begin{array}{c}63,50 \\
(23,92)\end{array}$ & $\begin{array}{c}63,97 \\
(24,51)\end{array}$ & $\begin{array}{c}68,97 \\
(23,79)\end{array}$ & $\begin{array}{c}70,68 \\
(22,95)\end{array}$ & $\begin{array}{c}70,46 \\
(22,47)\end{array}$ & $\begin{array}{c}71,90 \\
(22,66)\end{array}$ \\
\hline
\end{tabular}

*por mil habitantes

Em 2008, o PIB per capita médio da macrorregião de saúde era de R\$ 24 mil ( $D P=14,81)$, e em 2017, de R\$ 25 mil ( $D P=14,69)$. Quanto à taxa de equipamentos de diagnóstico por imagem em uso no SUS, foi identificado em análise descritiva aumento ao longo do tempo: em 2008 estava disponível $0,38(D P=0,19)$ e em 2017 avançou para $0,55(D P=0,23)$ equipamento para cada mil habitantes das macrorregiões de saúde (Tabela 1).

Quanto aos médicos no ano de 2008, foi identificado aumento no quantitativo, com leve queda para o ano de 2011 em relação a 2010: em 2008, apresentou 1,12 médico $(D P=0,55)$ para cada mil habitantes da macrorregião de saúde; em 2011, 1,20 (DP=0,60); e em 2017, 1,51 médico $(D P=0,68)$. No que se refere à cobertura pela $E S F$, também foi constatado aumento entre 2008 e 2017. No ano de 2008, 57,94\% (DP = 24,37) das macrorregiões de saúde no Brasil eram cobertas pela ESF, e, em 2017, esse valor foi de 71,90\% (DP =22,66) (Tabela 1).

A distribuição (Figura 2) do gasto médio per capita com diagnóstico de imagem em todas as microrregiões mostra um perceptível aumento gradual do gasto médio anual. Esse efeito pode ser percebido de forma global, pois o gasto médio brasileiro com diagnóstico de imagem aumentou de R\$3,26 em 2008 para R\$ 5,81 em 2017 (valores ajustados). Adicionalmente, esse aumento generalizado também pode ser percebido individualmente em cada microrregião, pois, integralizando as variações no gasto médio durante esse mesmo período, somente 4 das 104 microrregiões apresentaram diminuição nessa variável. Apesar disso, quando os dados são analisados aos pares em anos consecutivos, é notado que o número de microrregiões que aumentaram seu gasto vem diminuindo em relação ao número de microrregiões que diminuíram essa variável, com o caso notável dos anos 2014/2015, quando a proporção de microrregiões com diminuição de gasto foi maior do que aquelas com aumento nessa variável.

Foi realizada a análise de correlação entre os pares de todas as variáveis descritas anteriormente e individualmente para cada um dos anos compreendidos no período analisado neste estudo. Foi identificada correlação positiva de praticamente todas as variáveis explicativas com relação ao gasto per capita com diagnóstico de imagem de alta complexidade. Isso significa que um aumento de qualquer uma dessas variáveis está relacionado positivamente com o aumento do gasto per capita por imagem de alta complexidade. Por outro lado, a variável cobertura pela ESF manteve consistentemente para todo o período correlação negativa com essa mesma variável. 


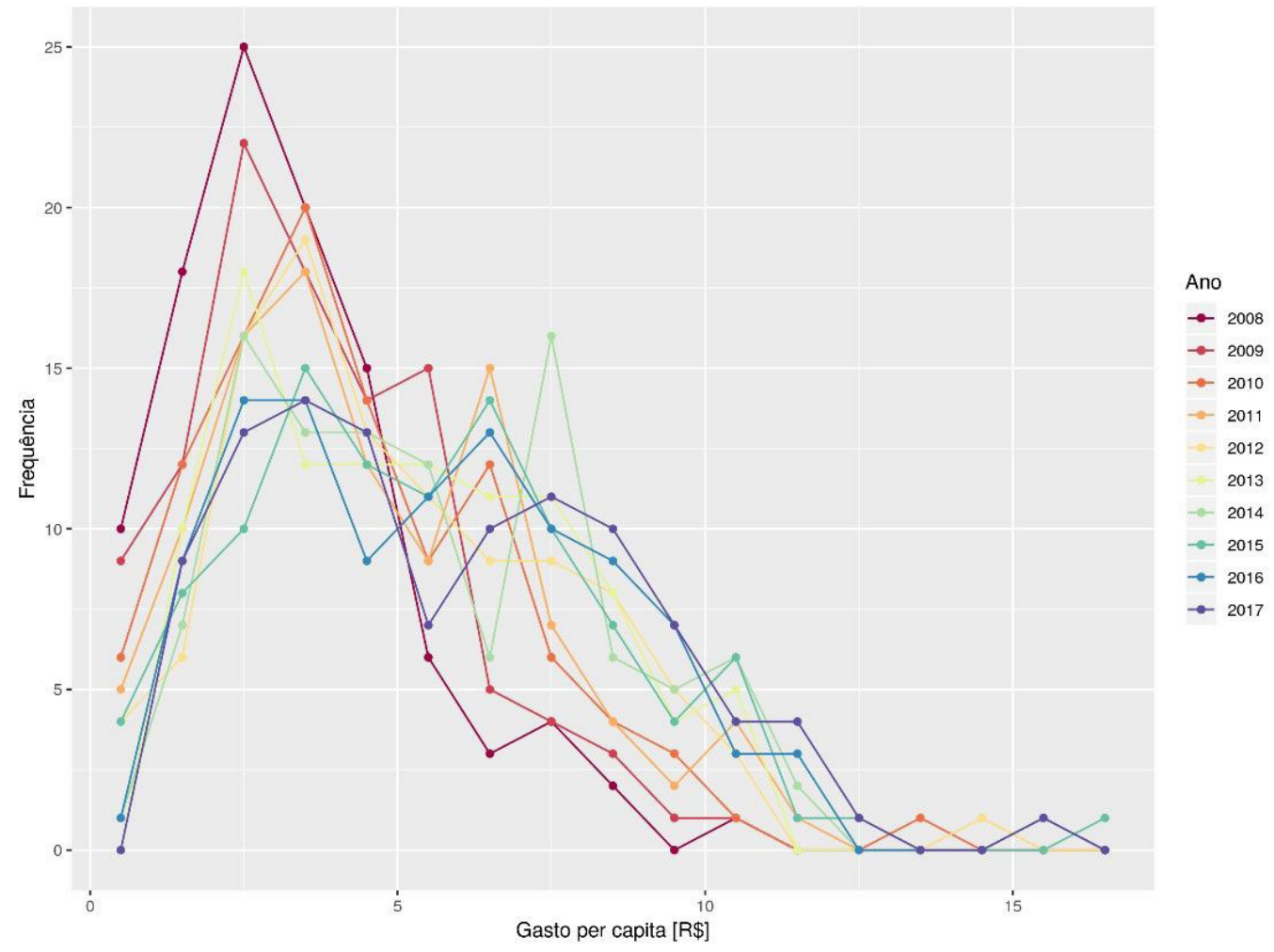

Figura 2. Histograma da distribuição da densidade (macrorregiões) dos gastos com diagnóstico por imagem de alta complexidade nos anos 2008 a 2017. Fonte: Datasus ${ }^{14}$ e IBGE ${ }^{33}$. Elaboração dos autores. Densidade: população das macrorregiões de saúde

A Tabela 2 apresenta os resultados do GLMM para a distribuição gama com ligação log, tendo como desfecho o gasto per capita com diagnóstico por imagem de alta complexidade ano a ano no Brasil. As estimativas dos parâmetros de dispersão foram de 0,005 para $\varnothing_{\boldsymbol{t}} \mathrm{e}$ de 0,164 para $\varnothing_{\boldsymbol{e}}$. O modelo mostra que a variável com maior impacto positivo nos gastos foi a taxa de médicos, seguida do PIB per capita; já com efeito negativo, a interação entre PIB e taxa de médicos. Por fim, macrorregiões com maiores índices de taxa de equipamentos e de cobertura pela ESF possuíam a tendência a diminuir esses gastos. Para interpretar as estimativas dos parâmetros do modelo (GLMM), foi aplicada a função exponencial na estimativa ( $\left.e^{\hat{\beta}}\right) \mathrm{e}$ comparada com o valor 1 . Assim, a cada médico a mais, havia um aumento de $141 \%$ nos gastos com diagnóstico por imagem; a cada aumento na unidade de taxa de equipamentos a mais, o gasto era reduzido em 67\%; a cada ano, o gasto aumentava em 3,9\%; a cada aumento de unidade do PIB, havia um aumento no gasto de 3\%; a cada aumento na porcentagem de cobertura pela ESF, ocorria uma redução de $0,8 \%$ no gasto. Porém, quando analisado o impacto das interações entre variáveis nos gastos com diagnóstico por imagem, são encontradas algumas mudanças nas interpretações. O aumento de PIB e de equipamentos provocou um crescimento de $1,4 \%$ nos gastos, enquanto o aumento de PIB e de médicos provocou uma redução de $0,7 \%$ nos gastos. Por fim, o aumento da taxa de equipamentos e de cobertura pela ESF provocou uma elevação de 1,1\% nos gastos.

\section{DISCUSSÃO}

Diante dos diversos fatores socioeconômicos, demográficos e de estrutura de serviços de saúde, em relação aos gastos com diagnóstico por imagem de alta complexidade, o presente estudo apontou heterogeneidade entre os territórios que compõem as macrorregiões de saúde no Brasil se perpetuando ao longo de 2008 a 2017. 
Tabela 2. Modelos lineares generalizados com efeitos mistos (GLMM) com distribuição gama e ligação log ajustados por máxima verossimilhança para os gastos com diagnóstico por imagem de alta complexidade

\begin{tabular}{lccccc}
\multicolumn{1}{c}{ Variáveis } & $\begin{array}{c}\text { Estimativa do } \\
\text { coeficiente }(\beta)\end{array}$ & $\begin{array}{c}\text { Limite } \\
\text { inferior } \\
\text { do IC }\end{array}$ & $\begin{array}{c}\text { Limite } \\
\text { superior } \\
\text { do IC }\end{array}$ & p-valor & Exp $(\beta)$ \\
\hline Intercepto & $-75,550$ & $-76,195$ & $-74,915$ & $<0,001$ & $\approx 0$ \\
\hline PIB per capita & 0,030 & 0,023 & 0,037 & $<0,001$ & 1,030 \\
\hline Taxa de equipamentos* & $-1,109$ & $-1,637$ & $-0,581$ & $<0,001$ & 0,330 \\
\hline Médicos* & 0,879 & 0,764 & 0,994 & $<0,001$ & 2,408 \\
\hline \% de cobertura pela ESF & $-0,0078$ & $-0,010$ & $-0,005$ & $<0,001$ & 0,992 \\
\hline Ano & 0,038 & 0,037 & 0,039 & $<0,001$ & 1,039 \\
\hline PIB vs taxa de equipamentos & 0,014 & 0,003 & 0,027 & 0,043 & 1,014 \\
\hline PIB vs médicos & $-0,017$ & $-0,021$ & $-0,013$ & $<0,001$ & 0,983 \\
\hline $\begin{array}{l}\text { Taxa de equipamentos vs } \\
\text { cobertura ESF }\end{array}$ & 0,011 & 0,006 & 0,016 & $<0,001$ & 1,011 \\
\hline
\end{tabular}

Legenda: PIB: produto interno bruto; ESF: Estratégia Saúde da Família.

*por mil habitantes

IC: Intervalo de Confiança de 95\%

Esse cenário retrata as desigualdades das macrorregiões de saúde, com destaque para as variáveis: PIB per capita, taxa de médicos e taxa de equipamentos em uso no SUS. Foi observado que os padrões de utilização de procedimentos de diagnóstico por imagem aumentaram ao longo do tempo, porém ainda permaneceram concentrados nos grandes centros brasileiros, os quais possuem melhores indicadores socioeconômicos ${ }^{15,16}$. Os gastos com diagnóstico por imagem nos anos de 2008 a 2010 apontaram para a desigualdade entre as macrorregiões de saúde, no entanto, no decorrer dos anos, ocorreu uma elevação contínua no número de macrorregiões que apresentavam maior gasto per capita com diagnóstico por imagem de alta complexidade, evidenciando diminuição na desigualdade do gasto.

Resultado semelhante foi encontrado no estudo de Albuquerque et al. ${ }^{17}$, que observaram grande evolução dos níveis de renda e oferta de serviços entre as 438 regiões de saúde durante a década de 2000 , com expressiva melhora nas condições socioeconômicas da população para os anos seguintes até 2016.

As mudanças nas desigualdades territoriais observadas a partir das regiões de saúde ao longo do tempo são explicadas por Albuquerque et al. ${ }^{17}$ e Barreto ${ }^{18}$ como reflexo de diversos fatores. O principal fator é a combinação de políticas sociais e econômicas, com crescimento da renda e da escolaridade, associada a estratégias regionais de desenvolvimento. Embora os avanços apontados por este e outros estudos sejam evidentes, ainda persistem as desigualdades regionais sociais e de saúde em todos os países, independentemente do grau de desenvolvimento alcançado ${ }^{19}$.

O gasto com diagnóstico por imagem de alta complexidade manteve correlação positiva com as variáveis PIB per capita, taxa de médicos, taxa de equipamentos em uso no SUS e população entre 2008 a 2017. Isso implica que macrorregiões com maior PIB per capita e maior taxa de médicos tendem a aumentar os gastos com diagnóstico por imagem de alta complexidade. A literatura ${ }^{20,21}$ tem destacado que as formas de financiamento das políticas públicas implementadas ao longo da história do país podem levar à concentração desordenada e à desarticulação entre os serviços, agravadas pelas diversidades socioculturais, desigualdade socioeconômica e singularidade epidemiológica que distinguem as regiões brasileiras. Além do mais, o prevalecimento do comportamento histórico da alocação de recursos, pautada 
na produção, e não nas necessidades, contribui para a manutenção das distribuições não equitativas dos recursos da saúde no Brasil22.

Entre as variáveis analisadas, a que se mostrou com mais impacto nos gastos foi a taxa de médicos, seguida do PIB per capita, inferência que tem gerado consequências na racionalização dos gastos. Hespanhol et al. ${ }^{23}$ e Norman e Tesser ${ }^{24}$ têm discutido a lógica da formação médica direcionada para a especialização e o quanto esta influencia o sistema, pois é no ambiente hospitalar de aprendizagem que historicamente o médico vem sendo formado. Este favorece a utilização dos exames de imagem como recursos preventivos, diagnósticos e terapêuticos, tornando-se conduta hegemônica para o esclarecimento de hipóteses diagnósticas ou por pressão de demanda dos pacientes.

A interação das variáveis PIB per capita e taxa de médicos mostrou redução do gasto per capita com diagnóstico por imagem. Nas regiões mais desenvolvidas economicamente, 0 incremento do gasto foi proporcionalmente menos influenciado com o aumento da riqueza. Para Barreto ${ }^{18}$, o estudo das tendências históricas das condições de saúde das populações continua sendo uma importante fonte de evidências, com destaque para as ciências econômicas e demográficas. No Brasil, o direito universal à saúde não se efetiva igualmente em todos os lugares, concretizando-se de maneira incompleta e seletiva no território em geral, seguindo as tendências regionais de concentração e escassez populacional, econômica e das modernizações técnicas, científicas e de informação, que caracterizam o meio geográfico ${ }^{17}$.

Embora seja essencial garantir o acesso às tecnologias avançadas de diagnóstico por imagem quando é indicado clinicamente, o crescimento substancial dessas tecnologias é um dos principais impulsionadores de gastos em assistência à saúde ${ }^{8,24}$.

Outra variável que mostrou impacto importante foi a taxa de equipamentos em uso no SUS. Essa variável possuiu correlação positiva com o gasto per capita com diagnóstico por imagem ao longo dos 10 anos estudados. Entretanto, ocorreu redução dos gastos nas macrorregiões com maior taxa de equipamentos em uso no SUS ao interagir com a cobertura pela ESF. Para Viegas e Penna ${ }^{25}$, a disponibilidade de recursos no SUS não acompanha o crescimento tecnológico, o que obriga a implantação de algum tipo de racionalização, com definição de prioridades, e leva em conta as questões éticas, ao apontar para práticas indutoras ou restritivas. Segundo Mitchell ${ }^{26}$, uma estratégia para diminuir a sobrecarga do uso de procedimentos de diagnóstico por imagem sinalizada pelo pagamento por produção indica que o seu uso deve ser fundamentado em diretrizes e protocolos de prática clínica para garantir que a aquisição de imagens avançadas seja usada apenas quando o diagnóstico clínico do paciente se justificar.

A implantação de protocolos e de diretrizes e a implementação de políticas públicas de saúde com foco na atenção primária podem ser a explicação para os resultados desta pesquisa em relação à cobertura pela ESF e o gasto per capita com diagnóstico por imagem de alta complexidade, a qual manteve correlação negativa entre 2008 e 2017. Esse resultado sugere o potencial da atenção primária à saúde, representada por cobertura pela ESF, na diminuição do gasto per capita com diagnóstico por imagem de alta complexidade.

Essa constatação foi percebida por alguns autores ${ }^{24,26}$ ao destacarem que já há evidências suficientes de que países cujos sistemas de saúde se organizam a partir dos princípios da atenção primária alcançam melhores resultados em saúde, menores custos, maior satisfação dos usuários e maior equidade, mesmo em situações de grande desigualdade social, como é o caso do Brasil. Pires et al. ${ }^{15}$, Spedo et al..$^{27}$ e Tesser e Poli Neto ${ }^{28}$ acrescentam que é necessário haver também mecanismos de coordenação assistencial com ordenamento dos fluxos e continuidade informacional que favoreçam a articulação da atenção primária à saúde com os serviços especializados.

Maia et al. ${ }^{29}$, Morimoto e Costa ${ }^{30}$ e Ceccon et al. ${ }^{31}$, que estudaram as internações por condições sensíveis à atenção primária (ICSAP) e a sua em relação com a ESF, concluíram que há influência na redução das taxas de ICSAP, o que se alinha com as evidências encontradas no presente estudo, quando foi apresentada a redução de gastos com diagnóstico por imagem na presença de maior cobertura pela ESF entre 2008 e 2017 nas macrorregiões de saúde Brasil.

Pesquisas recentes ${ }^{24,32}$ estudaram uma forma de prevenção desse avassalador uso da densidade tecnológica em todos os níveis da atenção à saúde. Segundo Martins ${ }^{32}$ e Norman 
e Tesser ${ }^{24}$, vários são os exemplos de situações em que os benefícios não se sobressaem, com destaque para o excesso de programas de rastreamento, muitos sem evidências; medicalização de fatores de risco; solicitação de exames complementares em demasia; excessos de diagnósticos, com rotulagem de quadros inexplicáveis ou não enquadráveis.

Portanto, os resultados obtidos neste estudo sugerem que, quando a comunidade não consegue acessar o serviço ou quando não encontra equipe apta para acolher as suas demandas, é elevada a solicitação de exames, o que pode gerar maiores gastos para o sistema de saúde. Grande parte do efeito encontrado neste estudo pode estar associado ao avanço tecnológico, expansão e disponibilidade de procedimentos de imagem diagnóstica, também relacionada ao aumento da demanda de procedimentos pelo usuário.

Ao mesmo tempo, a pesquisa indicou que existe uma concentração de médicos em locais com melhor infraestrutura socioeconômica, o que pode estar denotando a falta de incentivo na fixação de médicos nas regiões mais empobrecidas, visto que as necessidades de saúde devem ser ainda maiores nessas regiões.

Como limitações, pode haver imprecisões da base de dados utilizada e impossibilidade de inclusão da demanda reprimida, pois as análises verificaram apenas os gastos relacionados às transferências federais. Bancos de dados secundários são susceptíveis a erros e/ou fraudes. Assim, a partir do uso dessas bases de dados secundários disponíveis, é possível contribuir para a elaboração de estimativas e projeções que melhorem a qualidade dos dados para pesquisas e ações futuras.

Foi possível observar que a cobertura pela ESF se mostrou um importante indicador na redução dos gastos com diagnóstico por imagem de alta complexidade entre 2008 e 2017 nas macrorregiões de saúde no Brasil. Melhores condições socioeconômicas nas macrorregiões tendem a potencializar os gastos com diagnóstico por imagem de alta complexidade. Contudo, as regiões mais empobrecidas e menos atrativas para o grande capital são as que apresentam menor capacidade de investimentos na saúde, maiores dificuldades de atração e fixação de profissionais de saúde, menor capacidade de oferta assistencial pública e privada e maiores dificuldades no acesso aos procedimentos de imagem, especialmente por aquelas populações que não estão inseridas em grandes centros.

\section{REFERÊNCIAS}

1. Mitchell JM, LaGalia RR. Controlling the escalating use of advanced imaging: the role of radiology benefit management programs. Med Care Res Rev. 2009;66(3):339-51. http://dx.doi. org/10.1177/1077558709332055. PMid:19208823.

2. NHS England Analytical Services. NHS imaging and radiodiagnostic activity: 2013/14 release [Internet]. Wakefield: NHS England; 2014 [citado em 2018 jan 15]. Disponível em: https://www.england.nhs.uk/ statistics/wp-content/uploads/sites/2/2013/04/KH12-release-2013-14.pdf

3. Bhargavan $\mathrm{M}$, Sunshine JH. Utilization of radiology services in the United States: levels and trends in modalities, regions, and populations. Radiology. 2005;234(3):824-32. http://dx.doi.org/10.1148/ radiol.2343031536. PMid:15681686.

4. Carregaro RL, da Silva EN, van Tulder M. Direct healthcare costs of spinal disorders in Brazil. Int J Public Health. 2019;64(6):965-74. http://dx.doi.org/10.1007/s00038-019-01211-6. PMid:31093690.

5. Ferraz MB. Health care: the challenge to deal with uncertainty and value judgment. Cost Eff Resour Alloc. 2015;13(1):8. http://dx.doi.org/10.1186/s12962-015-0035-y. PMid:25949217.

6. Mendes EV. As redes de atenção à saúde. Brasília: Organização Pan-Americana da Saúde; 2011.

7. L'Organisation de Coopération et de Développement Économiques. OECD Health Statistics 2014. How does Brazil compare? [Internet]. 2014 [citado em 2018 maio 15]. Disponível em: http://www.oecd.org/ els/health-systems/Briefing-Note-BRAZIL-2014.pdf

8. Dilélio AS, Tomasi E, Thumé E, Silveira DS, Siqueira FCV, Piccini RX, et al. Padrões de utilização de atendimento médico-ambulatorial no Brasil entre usuários do Sistema Único de Saúde, da saúde suplementar e de serviços privados. Cad Saude Publica. 2014;30(12):2594-606. http://dx.doi. org/10.1590/0102-311x00118713. PMid:26247989. 
9. Instituto Brasileiro de Geografia e Estatística. Censo Demográfico [Internet]. Rio de Janeiro: IBGE; 2010 [citado em 2018 fev 16]. Disponível em: https://www.ibge.gov.br/estatisticas/sociais/trabalho/9662censo-demografico-2010.html?=\&t=resultados

10. Conselho Nacional de Secretários de Saúde. Assistência de média e alta complexidade no SUS. Brasília: CONASS; 2007.

11. Brasil. Ministério da Saúde. SIASUS - Sistema de Informações Ambulatoriais do SUS [Internet]. 2018 [citado em 2018 maio 15]. Disponível em: http://w3.datasus.gov.br/sia/index.php?area=0401

12. Brasil. Ministério da Saúde. Portaria no 2.848, de 21 de outubro de 2011. Aprova a Política Nacional de Atenção Básica, estabelecendo a revisão de diretrizes e normas para a organização da Atenção Básica, para a Estratégia Saúde da Família (ESF) e o Programa de Agentes Comunitários de Saúde (PACS). Diário Oficial da União. Brasília; 24 de outubro de 2011;

13. R:The R Project for Statistical Computing [Internet]. 2019 [citado em 2019 jun 28]. Disponível em: https:// www.r-project.org/

14. DATASUS. [Internet]. 2018 [citado em 2018 abr 30]. Disponível em: http://tabnet.datasus.gov.br

15. Pires MRGM, Göttems LBD, Martins CMF, Guilhem D, Alves ED. Oferta e demanda por média complexidade/ SUS: relação com atenção básica. Cien Saude Colet. 2010;15(Suppl 1):1009-19. http://dx.doi.org/10.1590/ S1413-81232010000700007. PMid:20640257.

16. Liebel G, Sá A Jr, Campos E, Loures F, Dias P, Chaoubah A. O diagnóstico por imagem no Brasil: um sistema, muitas realidades. J Bras Econ Saúde. 2018;10(3):291-7. http://dx.doi.org/10.21115/JBES.v10.n3.p291-7.

17. Albuquerque MV, Viana ALD, Lima LD, Ferreira MP, Fusaro ER, lozzi FL. Desigualdades regionais na saúde: mudanças observadas no Brasil de 2000 a 2016. Cien Saude Colet. 2017;22(4):1055-64. http://dx.doi. org/10.1590/1413-81232017224.26862016. PMid:28444033.

18. Barreto ML. Desigualdades em Saúde: uma perspectiva global. Cien Saude Colet. 2017;22(7):2097-108. http://dx.doi.org/10.1590/1413-81232017227.02742017. PMid:28723991.

19. Marmot M. The health gap: the challenge of an unequal world. London: Blomsbury; 2015.

20. Mendes AN, Marques RM. Os (Des)caminhos do financiamento do SUS. Saúde Debate. 2003;27(65):389404.

21. Mendes EV. 25 anos do Sistema Único de Saúde: resultados e desafios. Estud Av. 2013;27(78):27-34. http:// dx.doi.org/10.1590/S0103-40142013000200003.

22. Mendes Á, Leite MG, Marques RM. Discutindo uma Metodologia para a Alocação Equitativa de Recursos Federais para o Sistema Único de Saúde. Saude Soc. 2011;20(3):673-90. http://dx.doi.org/10.1590/S010412902011000300013.

23. Hespanhol AP, Couto L, Martins C. A medicina preventiva. Rev Port Clin Geral. 2008;24(1):49-64. http:// dx.doi.org/10.32385/rpmgf.v24i1.10462.

24. Norman AH, Tesser CD. Quaternary prevention: a balanced approach to demedicalisation. Br J Gen Pract. 2019;69(678):28-9. http://dx.doi.org/10.3399/bjgp19X700517. PMid:30591604.

25. Viegas SMF, Penna CMMO. SUS é universal, mas vivemos de cotas. Cien Saude Colet. 2013;18(1):181-90. http://dx.doi.org/10.1590/S1413-81232013000100019. PMid:23338508.

26. Mitchell JM. Utilization trends for advanced imaging procedures: evidence from individuals with private insurance coverage in California. Med Care. 2008;46(5):460-6. http://dx.doi.org/10.1097/ MLR.0b013e31815dc5ae. PMid:18438193.

27. Spedo SM, Pinto NRS, Tanaka OY. O difícil acesso a serviços de média complexidade do SUS: o caso da cidade de São Paulo, Brasil. Physis Rev Saúde Coletiva. 2010;20(3):953-72. http://dx.doi.org/10.1590/ S0103-73312010000300014.

28. Tesser CD, Poli Neto P. Atenção especializada ambulatorial no Sistema Único de Saúde: para superar um vazio. Cien Saude Colet. 2017;22(3):941-51. http://dx.doi.org/10.1590/1413-81232017223.18842016. PMid:28301001.

29. Maia LG, Silva LA, Guimarães RA, Pelazza BB, Pereira ACS, Rezende WL, et al. Internações por condições sensíveis à atenção primária: um estudo ecológico. Rev Saude Publica. 2018;53:02. http://dx.doi. org/10.11606/S1518-8787.2019053000403. PMid:30652775.

30. Morimoto T, Costa JSD. Internações por condições sensíveis à atenção primária, gastos com saúde e Estratégia Saúde da Família: uma análise de tendência. Cien Saude Colet. 2017;22(3):891-900. http:// dx.doi.org/10.1590/1413-81232017223.27652016. PMid:28300996. 
31. Ceccon RF, Meneghel SN, Viecili PRN. Hospitalization due to conditions sensitive to primary care and expansion of the Family Health Program in Brazil: an ecological study. Rev Bras Epidemiol. 2014;17(4):96877. http://dx.doi.org/10.1590/1809-4503201400040014. PMid:25388495.

32. Martins C, Godycki-Cwirko M, Heleno B, Brodersen J. Quaternary prevention: reviewing the concept. Eur J Gen Pract. 2018;24(1):106-11. http://dx.doi.org/10.1080/13814788.2017.1422177. PMid:29384397.

33. Instituto Brasileiro de Geografia e Estatística. Indicadores do desenvolvimento brasileiro: 2001-2012. Rio de Janeiro: Ministério do Planejameneto, Orçamento e Gestão, 2012. 